\title{
Effect of Problem Solutions on Students' Reasoning Patterns on Conceptual Physics Problems
}

\author{
Xian Wu, Tianlong Zu, Elise Agra and N. Sanjay Rebello \\ Department of Physics, Kansas State University, 116 Cardwell Hall, Manhattan, KS 66505-2601
}

\begin{abstract}
Conceptual knowledge is only one part of what students need to learn to solve problems. Knowing how to reason through a problem is also an important skill. In this paper, we study the effects of a computer-based training on students' reasoning on introductory physics problems. Participants solved three sets of conceptual problems, each of them containing three training problems with solutions followed by one near transfer problem and one far transfer problem. We analyzed students' verbal answers to elucidate the reasoning resources that they activated to construct the different explanations to the problems. We found that the students changed their reasoning when presented with solutions to training problems and later posed a transfer problem. Our results provide insights into students' activation of their resources and the procedure they used to construct their reasoning in response to the training problems.
\end{abstract}

Keywords: problem solving, resources, multimedia learning, transfer

PACS: 01.40.Fk

\section{INTRODUCTION}

Multimedia instruction in physics is increasingly ubiquitous. Multimedia instruction often relies on increasing the salience of relevant information. ${ }^{1}$ Our previous research showed that manipulating the perceptual salience alone cannot influence students' visual attention or responses. ${ }^{2}$ Here we provide students with a complete solution to a problem within which we enhanced the perceptual salience of relevant information. We investigated changes in students' answers and reasoning after being presented such problem solutions.

\section{THEORETICAL FRAMEWORK}

A resource is a piece of knowledge that a student uses to answer a question. ${ }^{3}$ Student responses were characterized in terms of the reasoning resources. Students have multiple stances toward knowledge and learning available. They can potentially use different resources in answering questions having the same scientific explanation. Our research questions were:

1. What resources do students activate while solving the problems in our study?

2. How would the provided solution affect students' resource activation?

\section{METHODOLOGY}

All participants $(\mathrm{N}=42)$ were recruited from 74 students, mostly sophomores and juniors, enrolled in a conceptual physics class for non-science students at a Midwestern university. Students received 50 points credit for participating in a 1-hour interview toward the total points (1000) in the course. Out of these, 45 points replaced the three lowest homework scores. The remaining 5 points were extra credit. The interview contained three problem sets some of which were based on questions from the FCI. ${ }^{4}$ Each problem set consisted of three training problems (e.g. Fig. 1), a near transfer problem (e.g. Fig. 3), and a far transfer problem (e.g. Fig. 4) presented on a computer screen.

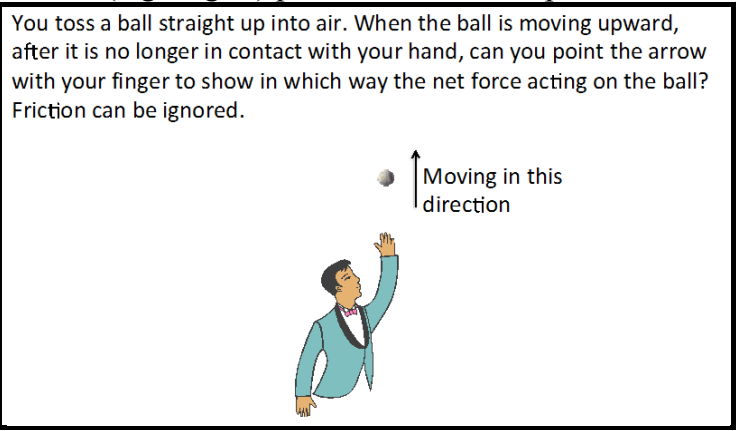

FIGURE 1. Example of a training problem.

After verbally answering each training problem students were provided a solution on a separate slide (see Fig. 2). The solution slide contained a perceptually salient 'take-home' message based on the Multimedia Learning Theory. ${ }^{5}$ The take-home message contained the main concept that students would need to answer the transfer problems. In Fig. 2 the take-home message is "The direction of net force is not necessarily the same as the direction of motion." 
ם

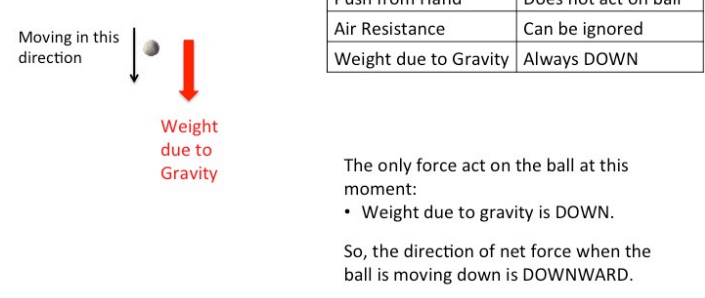

Direction of net force is not necessarily the same as the direction of motion.

FIGURE 2. Solution to the training problem in Fig 1.

Various snapshots of a springboard diver are shown. What is the direction of net force acting on the diver at position 2 in the diagram? Friction can be ignored.

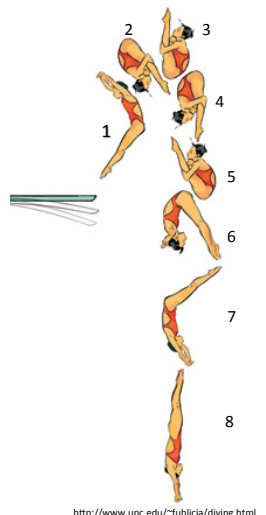

FIGURE 3. Near transfer problem corresponding to Fig 1.

You are shown a snapshot of the car that was given a push up a ramp. The direction of the net force acting on the car is also shown in the picture. Based on the information provided about this snapshot, can you tell whether the car is moving, and if so in what direction it is moving? Friction can be ignored.

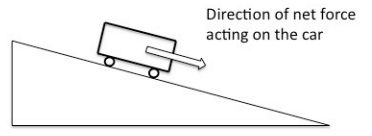

FIGURE 4. Far transfer problem corresponding to Fig 3.

After answering all problems in a set, students were asked to talk about similarities and differences between the problems and the provided solutions and their own solutions. The sequence of problem sets was randomized to control for order effects. The videorecorded interviews were analyzed based on a phenomenographic analysis.

In the next section we discuss one representative problem set. First, we describe the categories of reasoning resources. Then we discuss the patterns of changing resources. The coding scheme of producing these categories was not pre-designed. Rather, the codes emerged from student responses by analyzing the responses for their reasoning elements using the resources framework. Two coders coded student responses separately. The inter-rater reliability between the two coders was $87.5 \%$ before discussion and $90.9 \%$ after discussion.

\section{RESULTS}

\section{Activated Resources}

Students used the following resources to talk about the net force direction on the tossed ball in Fig. 1.

Motion with Force (MwF): The ball is going up, so the net force has to be going up. It seems that students activated this resource based on the surface feature "ball is moving upward" in the question. So they associated the net force direction to the ball's direction of motion consistent with a widely reported misconception. ${ }^{6}$

Motion against Force (MaF): The net force opposes the object's motion. Students who seemed to activate this resource often referred to "air resistance" but failed to explain how it related to the net force. Further, these students persisted in referring to air resistance even when reminded that "friction can be ignored."

Impetus Force (IF): Net force is in the direction of the push that caused the object to move (hand or springboard). This is similar to a previously reported misconception. ${ }^{6}$ Interestingly, students not only used this resource to reason about the object moving up, some even used it when the object moved down.

Gravity Downward (GD): Some students stated that net force was downward due to "gravity." but were unable to provide a supporting reason. After viewing the solution slide (Fig 4.) students were more likely to refer to gravity on the transfer problems. However, their responses to follow-up questions revealed their notion that gravity and net force was the "same thing."

Take-Home Message (THM): Some students activated a resource based on the take-home message in the solution slide i.e. "Direction of net force is not necessarily the same as the direction of motion."

Motion and Force (M\&F): Some students insisted that they needed to know "how large is the push" to determine the car's moving direction in the far transfer problem. Interestingly they did not make such requests while answering any previous problems.

No motion No force (NN): Some students reasoned "the ball is not moving at its highest point so there is no net force" in the second training problem.

Previous Solution (PS): Some students noticed similarities between the training and transfer problems and seemed to overgeneralize these similarities stating for instance that "the answers are all downward due to gravity" and provided no further supporting reason.

\section{Resource Activation Change Patterns}

Five types of resource change patterns emerged from analyzing students' answers across the entire 
problem set: Using the Take-home Message (explicitly using the highlighted take-away message provided in the trial problem solution as a resource in successive problems); Incomplete Learning (suppressing some unproductive resources and activating productive ones on the successive problem, but not necessarily based on the take-home message); Trial and Error (noticing that their answers were wrong after seeing the solution and activating different albeit unproductive resources); Failure to Transfer (activating productive resources after viewing the solution on the training problems but failing to reactivate them on the transfer problems); Consistency (activating similar resources on almost all problems). Given the limited space here, we first discuss Using the Take-home Message since it is indicative of learning and transfer and later discuss the overarching trends in resource change.

\section{Using the Take-Home Message}

Here is an example of a student who activated unproductive resources on the first training problem and gradually began to activate productive resources based on the take-home message in the solution:

$\left(1^{\text {st }}\right.$ training problem - Resource: $\left.\mathrm{MwF}\right)$

S: OK, I think the net force is moving up.

I: And why is that?

S: Because that's the direction you were sending the ball and left your hands and it doesn't say anything about the point it hit, like the highest point it could go and turning around. So I'm assuming the net force is still on its way, straight up.

$\left(2^{\text {nd }}\right.$ training problem - Resources: MwF then $\left.\mathrm{NN}\right)$

S: Straight down.

I: And what's your reason?

$\mathrm{S}$ : Because after it reaches the highest point there is only one direction it can only go and that's down. Therefore, the force must be acting down.

I: Well, but the question is asking at this moment, at the moment that the ball is at its highest point.

S: OK, then maybe there is no net force. At the highest point, it's like, zero, for seconds, and there is no net force.

I: OK, if you say there is no net force, what's your reason for that?

S: Because... it's like the turnaround or its breaking point and for split second, it's not moving up nor down and so at that split second, there is no force.

$\left(3^{\text {rd }}\right.$ training problem - Resource: GD)

S: Straight down.

I: And what's your reason for that?

$\mathrm{S}$ : The only force acting on the ball is gravity and gravity pulls it down.

(Near transfer problem - Resources: GD, THM)

$\mathrm{S}$ : Because the net force doesn't necessarily relate to the direction that the object is travelling. So even though on 2 she was going higher before she actually falls, gravity is the force and gravity is pulling down.

(Far transfer - Resource: THM)

S: You cannot tell whether car is moving or not.

I: Why is that?

S: Because the arrow doesn't denote movement, it denotes the net force. And it's gonna like the ball when it's at its highest point in the air. There is still net force pointing down, regardless whether the ball is at its highest peak point, and it's like stop for split second or it's falling. It's going down, so this arrow gonna denote net force whether it's actually moving or whether at that split second, steady still.

This student seemed to attend to the highlighted information pertaining to gravity starting with the $3^{\text {rd }}$ training problem. There are several reasons for this prevalent trend. First, the information "weight due to gravity" denoted by the arrow in the diagram and its position was very close to the diagram. According to Multimedia Learning Theory, ${ }^{5}$ proximity of text and pictures conveying the same information facilitates learning. The proximity of the red downward arrow to the red text facilitates students to attend to the takehome message. Second, gravity is a very familiar term for these students and so they face a low cognitive load barrier to integrate this information with their prior knowledge. Finally, this student incorporated information from the solution slide on the far transfer problem, namely the THM resource, most likely due to its perceptual salience due to the red text.

\section{Overarching Trends in Resource Activation}

The above example shows that perceptual salience can draw students' attention to facilitate activation of productive resources on a far transfer problem. However, a vast majority of students failed to activate the resource in productive way. Figure 5 presents the trends of changes in resource activation. No matter what resources students activated in the first training problem, the majority of them (26 of 42) activated GD on the $2^{\text {nd }}$ training problem. The number of students who activated GD increased from the $2^{\text {nd }}$ to the $3^{\text {rd }}$ training problems (33 of 42). About $1 / 3$ of the students activated GD in the third training problem but changed to $\mathrm{MwF}$ on the near transfer problem, indicating that changing the problem's surface feature affects students' resource activation. Ten students still activated the GD resource for the far transfer problem's answer, although gravity is seemingly unrelated to the question. However, a vast majority of students (28 of 42) activated the MwF resource on the transfer problems indicating a failure to transfer activation of the THM resource on the transfer problem. 


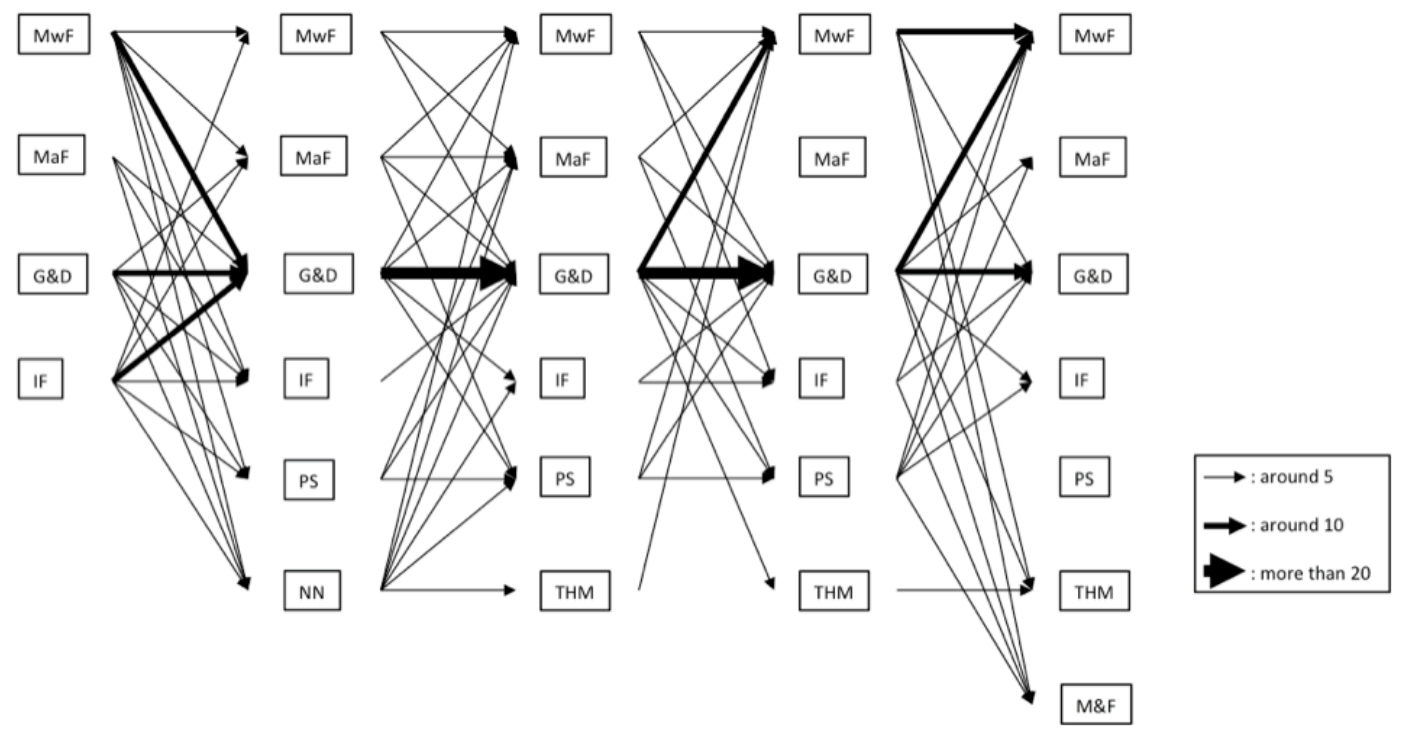

FIGURE 2. Resource activation change going from the $1^{\text {st }}, 2^{\text {nd }}$, and $3^{\text {rd }}$ training problems and the near and far transfer problems.

\section{CONCLUSIONS}

We have investigated whether providing complete solutions that highlight the important conceptual elements needed to solve a problem can affect students' resource activation on a similar problem as well as on a near and far transfer problem. We found that students activated a variety of resources while solving the initial problem. After being presented with solutions, their responses began to converge toward particular resources that were consistent with the perceptually salient take-home message provided on the slides. However, when presented with the transfer problems their resource activation changed significantly and was not necessarily consistent with the take-home message on the solution slides.

These results suggest that for the most part the takehome message on solution slides, even though it is made perceptually salient, does not have a significant impact on students' resource activation on the transfer problems. There are two possible reasons that we can suggest. One is that perceptual salience, which primarily controls bottom-up cognitive processes, alone cannot facilitate students to activate appropriate resources on transfer problems. This is because when the visually salient message is no longer present, such as on the transfer problem, the top-down processes which are based on students' domain knowledge i.e. inappropriate resources, dominates. The other reason is that the design of solution slides does not facilitate students to articulate the differences between their reasoning and correct reasoning. Thus, students tend to selectively attend to information on the solution slides that is consistent with the prior knowledge rather than focusing on that information which is inconsistent with their prior knowledge. In other words, the topdown processes that are controlled by the students' prior knowledge seem to dominate bottom-up processes that are driven by visual salience. Therefore the resource activation patterns on the transfer problems are governed more by students' prior knowledge than the information provided on the solution slides. This misunderstanding could be another reason why the solution slides did not impact significantly students' resource activation on the transfer problems.

\section{ACKNOWLEDGMENTS}

This work is supported in part by the U.S. National Science Foundation under Grant No. 1348857. Opinions expressed are of the authors and not of the Foundation.

\section{REFERENCES}

1. Z. Chen, Ph.D. Thesis, University of Illinois, 2013.

2. A. M. Madsen, Ph. D. Thesis, Kansas State University, 2012.

3. D. Hammer, Phys. Educ. Res, Am. J. Phys. 68, pp. S52S59 (2000).

4. D. Hestenes, M. Wells, and G. Swackhamer, The Physics Teacher 30(3), 141-158 (1992).

5. R. Mayer, The Cambridge Handbook of Multimedia Learning (Cambridge University Press, New York, 2005).

6. R. Gunstone and M. Watts, Children's Ideas in Science, (Open University Press, Buckingham, 1985). 